\title{
Sudden Cardiac Death and Idiopathic Ventricular Arrhythmias
}

\author{
Lennart de Vries, Zsuzsanna Kis, Sing-Chien Yap \\ Department of Cardiology, Erasmus Medical Center, Rotterdam, the Netherlands
}

\section{ABSTRACT}

Ventricular arrhythmias (VAs) in patients without structural heart disease can be found in a significant portion of the general population. The prognosis of patients with idiopathic VA is usually favorable and patients are often asymptomatic. However, sudden cardiac death (SCD) as a consequence of idiopathic VA has been reported. The aim of this review is to present an overview of the association between idiopathic VA and SCD.

Keywords: idiopathic ventricular arrhythmia, sudden cardiac death, malignant

\section{ARTICLE HISTORY}

Received: 2 September, 2015

Accepted: 4 November, 2015

CORRESPONDENCE

Sing-Chien Yap

Thoraxcenter, Department of Clinical Electrophysiology

Erasmus MC

Postbus 2040, 3015 CE Rotterdam,

The Netherlands

'sGravendijkwal 230, Room BA-579

Tel: +31-10-7033991

Fax: $+31-10-7034420$

Email: s.c.yap@erasmusmc.nl

\section{INTRODUCTION}

Premature ventricular contractions (PVCs) and ventricular tachycardias (VTs) that are observed in patients without overt structural heart disease are considered idiopathic ventricular arrhythmias (VA). Idiopathic VA usually originates from the outflow tract region [1]. These arrhythmias are generally considered benign, although in some cases they can lead to severe symptoms and/or cardiomyopathy [2-8]. It is important to rule out conditions which may be associated with malignant VA originating from the outflow tract, such as arrhythmogenic right ventricular cardiomyopathy (ARVC), Brugada syndrome or catecholaminergic polymorphic VT. After excluding these potential malignant causes of VA, some patients with idiopathic VA are at risk of SCD (although this risk is rare). In this review we aim to summarize the association between idiopathic VA and SCD.

\section{MALIGNANT IDIOPATHIC VA}

The most common form of idiopathic VA is an outflow tract VT which usually originates from the right ventricular outflow tract (RVOT). Most forms of outflow tract VTs are adenosine-sensitive and are thought to be mediated by catecholamine-induced, delayed after depolarizations (DADs)-induced triggered activity [9-11]. Although the prognosis of outflow tract ectopy is considered to be good [12-17], rapid polymorphic VT (PVT) and/or ventricular 
fibrillation (VF) are occasionally initiated by RVOT PVCs $[18,19]$.

There is a growing body of evidence that very short coupled PVC's may initiate idiopathic VF $[19,20]$. These close coupled beats fall in the vulnerable phase of ventricular activation and usually originate from the distal Purkinje system. Haissaguerre et al. described a case series of 27 patients with idiopathic VF initiated by a short-coupled PVC [21]. Most patients had initiating PVCs from the distal Purkinje conduction system. However, 4 patients had PVCs originating from the RVOT, which is interesting because ventricular ectopy from the RVOT is usually considered benign. The sources of the Purkinje beats were approximately equally distributed between both ventricles. Patients with VF initiated from the Purkinje system were older and had more episodes of VF and polymorphic beats, and $26 \%$ had a familial history of SCD. Other groups have also described RVOT PVCs initiating PVT/VF $[18,19]$. The exact incidence of malignant idiopathic VA is not known. Most cases are described in case reports or case series with a highly selected population (i.e. patients undergoing electrophysiologic study for treatment of PVCs initiating idiopathic VF). One report demonstrated a prevalence of $16 \%$ of spontaneous PVT/VF in patients referred for radiofrequency catheter ablation of VA arising from the RVOT [18]. This reflects referral bias, as patients with PVT are more likely to be referred for catheter ablation.

\section{RECOGNIZING MALIGNANT IDIOPATHIC VA}

In general, the coupling interval of PVCs triggering malignant VT or VF is shorter than PVC's triggering benign VT [19]. Especially in those with idiopathic VF, the initiating PVC usually falls on the peak of the T-wave [21]. In the study of Viskin et al., the coupling intervals of the initiating PVC in those with idiopathic VF, malignant RVOT VT, and benign RVOT VT was $300 \pm 40 \mathrm{~ms}, 340 \pm 30 \mathrm{~ms}$, and $427 \pm 76 \mathrm{~ms}$, respectively [19]. However, considerable overlap exists and there is not a clear cutoff value that could differentiate malignant and benign PVCs. Igarashi et al. suggested that a prematurity index (the coupling interval divided by the QT interval of the preceding sinus complex) $<0.73$ can identify malignant PVCs with a sensitivity of $91 \%$ and a specificity of $44 \%$ [22].

Noda et al. described the clinical characteristics of 16 patients with RVOT PVCs initiating VF and/or polymorphic VT (PVT) [18]. Interestingly, the coupling interval of the PVCs triggering PVT/VF was long, which is in contrast with the short-coupled Purkinje system related PVCs as described earlier. Furthermore, 11 patients (69\%) showed pre-syncope or syncope as the first symptom, the remain- ing patients had only palpitations due to PVCs or monomorphic VT as a first symptom. In comparison to patients with benign RVOT VT, patients with VF/PVT more often had a history of syncope (69\% versus 18\%) and shorter cycle length of non-sustained VT on previous Holter recordings ( $245 \pm 28 \mathrm{~ms}$ versus $328 \pm 65 \mathrm{~ms}$ ). This highlights the necessity for careful follow-up of patients with a history of syncope and frequent RVOT PVC's.

Another study compared patients with benign and malignant outflow tract VTs [23]. Patients with malignant outflow tract VT were defined as having syncope, aborted SCD or VF. On analysis of non-sustained VT in these patients, the second coupling interval of NSVT beats was significantly shorter in the malignant group $(313 \pm 58$ ms versus $385 \pm 83 \mathrm{~ms}$ ), whereas the first coupling interval of NSVT was similar between groups. Furthermore, the malignant group frequently had more than 1 focus of VT, whereas the benign group showed only a single focus. Thus a short second coupling interval of NSVT or demonstration of multiple VT origins may help identify the malignant form of outflow tract VT [23].

\section{MANAGEMENT OF MALIGNANT VA}

Successful radiofrequency catheter ablation of PVCs initiating idiopathic VF has been described by multiple groups [22,24-27]. Knecht et al. reported one of the largest series of patients with idiopathic VF treated with radiofrequency catheter ablation [27]. Thirty-eight patients from 6 different centers underwent ablation of idiopathic VF initiated by short coupled PVCs. PVCs arose from the right Purkinje system in 16 patients, the left Purkinje system in 14 patients, from both the left and right Purkinje system in 3 patients, and in the myocardium in 5 patients $(80 \%$ RVOT). During a median follow-up of 63 months, $18 \%$ of patients experienced VF recurrence. A majority of these patients underwent repeated ablation without any recurrence. Thus, radiofrequency catheter ablation seems to be effective in the management of patients with idiopathic VF initiated by short coupled PVCs.

Noda et al. performed radiofrequency ablation in 16 patients with RVOT PVCs initiating spontaneous PVT/VF [18]. Ablation was successful in 13 patients and partially successful in 3 patients. During a follow-up of 54 months there were no recurrences of syncope, VF or cardiac arrest in this group.

\section{CONCLUSION}

Idiopathic VA, usually RVOT ectopy, is common and has a benign prognosis. However, RVOT ectopy may cause rapid 
polymorphic VT/VF in rare cases, potentially leading to SCD. Appropriate efforts should be made to rule out conditions known to be associated with malignant VA from the outflow tract, especially ARVC and Brugada syndrome. Early radiofrequency catheter ablation should be offered to patients with high risk factors such as history of syncope, very fast VT (>230 bpm), and PVCs with short coupling interval.

\section{REFERENCES}

1. Brooks R, Burgess JH. Idiopathic ventricular tachycardia. A review. Medicine (Baltimore). 1988;67:271-294.

2. Pytkowski M, Maciag A, Jankowska A, et al. Quality of life improvement after radiofrequency catheter ablation of outflow tract ventricular arrhythmias in patients with structurally normal heart. Acta Cardiol. 2012;67:153-159.

3. Chugh SS, Shen WK, Luria DM, Smith HC. First evidence of premature ventricular complex-induced cardiomyopathy: a potentially reversible cause of heart failure. J Cardiovasc Electrophysiol. 2000;11:328-329.

4. Kanei Y, Friedman M, Ogawa N, Hanon S, Lam P, Schweitzer P. Frequent premature ventricular complexes originating from the right ventricular outflow tract are associated with left ventricular dysfunction. Ann Noninvasive Electrocardiol. 2008;13:81-85.

5. Yao J, Xu J, Yong YH, Cao KJ, Chen SL, Xu D. Evaluation of global and regional left ventricular systolic function in patients with frequent isolated premature ventricular complexes from the right ventricular outflow tract. Chin Med J (Engl). 2012;125:214-220.

6. Kennedy HL, Whitlock JA, Sprague MK, Kennedy LJ, Buckingham TA, Goldberg RJ. Long-term follow-up of asymptomatic healthy subjects with frequent and complex ventricular ectopy. N Engl J Med. 1985;312:193-197.

7. Zweytick B, Pignoni-Mory P, Zweytick G, Steinbach K. Prognostic significance of right ventricular extrasystoles. Europace. 2004;6:123-129.

8. Gaita F, Giustetto C, Di Donna P, et al. Long-term follow-up of right ventricular monomorphic extrasystoles. J Am Coll Cardiol. 2001;38:364-370.

9. Lerman BB, Belardinelli L, West GA, Berne RM, DiMarco JP. Adenosine-sensitive ventricular tachycardia: evidence suggesting cyclic AMP-mediated triggered activity. Circulation. 1986;74:270-280.

10. Lerman BB. Response of nonreentrant catecholaminemediated ventricular tachycardia to endogenous adenosine and acetylcholine. Evidence for myocardial receptor-mediated effects. Circulation. 1993;87:382-390.

11. Lerman BB, Stein K, Engelstein ED, Battleman DS, Lippman N, Bei D, Catanzaro D. Mechanism of repetitive monomorphic ventricular tachycardia. Circulation. 1995;92:421-429.

12. Froment R, Gallavardin L, Cahen P. Paroxysmal ventricular tachycardia; a clinical classification. Br Heart J. 1953;15:172-178.

13. Rahilly GT, Prystowsky EN, Zipes DP, Naccarelli GV, Jackman
WM, Heger JJ. Clinical and electrophysiologic findings in patients with repetitive monomorphic ventricular tachycardia and otherwise normal electrocardiogram. Am J Cardiol. 1982;50:459-468.

14. Buxton AE, Waxman HL, Marchlinski FE, Simson MB, Cassidy $\mathrm{D}$, Josephson ME. Right ventricular tachycardia: clinical and electrophysiologic characteristics. Circulation. 1983;68:917927.

15. Lemery R, Brugada P, Bella PD, Dugernier T, van den Dool A, Wellens HJ. Nonischemic ventricular tachycardia. Clinical course and long-term follow-up in patients without clinically overt heart disease. Circulation. 1989;79:990-999.

16. Mont L, Seixas T, Brugada P, et al. The electrocardiographic, clinical, and electrophysiologic spectrum of idiopathic monomorphic ventricular tachycardia. Am Heart J. 1992;124:746-753.

17. Ohe T, Shimomura K, Aihara N, et al. Idiopathic sustained left ventricular tachycardia: clinical and electrophysiologic characteristics. Circulation. 1988;77:560-568.

18. 18.Noda T, Shimizu W, Taguchi A, et al. Malignant entity of idiopathic ventricular fibrillation and polymorphic ventricular tachycardia initiated by premature extrasystoles originating from the right ventricular outflow tract. J Am Coll Cardiol. 2005;46:1288-1294.

19. Viskin S, Lesh MD, Eldar M, et al. Mode of onset of malignant ventricular arrhythmias in idiopathic ventricular fibrillation, J Cardiovasc Electrophysiol. 1997;8:1115-1120.

20. Leenhardt A, Glaser E, Burguera M, Nürnberg M, MaisonBlanche P, Coumel P. Short-coupled variant of torsade de pointes. A new electrocardiographic entity in the spectrum of idiopathic ventricular tachyarrhythmias. Circulation. 1994;89:206-215.

21. Haïssaguerre M, Shoda M, Jaïs P, et al. Mapping and ablation of idiopathic ventricular fibrillation, Circulation. 2002;106:962967.

22. Igarashi $M$, Tada $H$, Kurosaki $K$, et al. Electrocardiographic determinants of the polymorphic QRS morphology in idiopathic right ventricular outflow tract tachycardia. J Cardiovasc Electrophysiol. 2012;23:521-526.

23. Kim YR, Nam GB, Kwon CH, et al. Second coupling interval of non-sustained ventricular tachycardia to distinguish malignant from benign outflow tract ventricular tachycardias. Heart Rhythm. 2014;11:2222-2230.

24. Betts TR, Yue A, Roberts PR, Morgan JM. Radiofrequency ablation of idiopathic ventricular fibrillation guided by noncontact mapping. J Cardiovasc Electrophysiol. 2004;15:957-959.

25. Kataoka M, Takatsuki S, Tanimoto K, Akaishi M, Ogawa S, Mitamura H. A case of vagally mediated idiopathic ventricular fibrillation., Nat Clin Pract Cardiovasc Med. 2008;5:111-115.

26. Naik N, Juneja R, Sharma G, Yadav R, Anandraja S. Malignant idiopathic ventricular fibrillation "cured" by radiofrequency ablation. J Interv Card Electrophysiol. 2008;23:143-148.

27. Knecht S, Sacher F, Wright M, et al. Long-term follow-up of idiopathic ventricular fibrillation ablation: a multicenter study. J Am Coll Cardiol. 2009;54:522-528. 